\title{
Erratum to: "A Variational Principle for Problems of Steady-State Ground Water Flow with a Free Surface" [Fluid Dynamics 50, 543 (2015)]
}

\author{
A. Yu. Belyaev and I. O. Yushmanov \\ Water Problems Institute, Russian Academy of Sciences, \\ ul. Gubkina 3, Moscow, 119333 Russia \\ e-mail:beliaev@aqua.laser.ru \\ Received September 3, 2015
}

DOI: $10.1134 / \mathrm{S} 0015462815050142$

On page 543 A. I. Belyaev should be read A. Yu. Belyaev. 